\title{
Current Considerations for Breast Reconstruction in Breast Cancer Patients
}

\author{
Keli Kolegraff • Benjamin Moosavi • Albert Losken
}

Published online: 21 December 2012

(C) Springer Science+Business Media New York 2012

\begin{abstract}
In the next year, approximately 250,000 women will be diagnosed with breast cancer in the United States [1]. The majority of these women will be recommended partial or total mastectomy as a component of their cancer treatment. The goal of breast reconstruction is to rebuild and/or reshape the breast mound to correct the mastectomy defect for physical and psychological reasons. There are a number of reconstructive options available to patients who desire breast reconstruction, including implant-based procedures, autologous tissue reconstructions, or a combination. Additionally, oncoplastic procedures are a recent advance in the field of breast-conserving therapies that further refines our ability to resect breast cancer while preserving the form of the breast. Because planning the reconstruction depends on the type of resection performed and the amount of tissue remaining after mastectomy, the breast surgeon, reconstructive surgeon, and the patient collectively should discuss reconstructive goals and how these can be achieved after the patient's oncologic surgery.
\end{abstract}

Keywords Breast reconstruction - Oncoplastic reduction, Autologous flap · Mastectomy · Breast cancer · TRAM, Breast implants $\cdot$ Managing breast problems

\section{Introduction}

Breast cancer is the most common malignancy affecting women in the United States; it is estimated that one in eight

\author{
K. Kolegraff $\cdot$ B. Moosavi $\cdot$ A. Losken $(\triangle)$ \\ Division of Plastic and Reconstructive Surgery, \\ Emory University School of Medicine, Atlanta, GA 30322, USA \\ e-mail: alosken@emory.edu \\ K. Kolegraff \\ e-mail: kkolegr@emory.edu \\ B. Moosavi \\ e-mail: ben.moosavi@emory.edu
}

will develop breast cancer in their lifetime [1]. The mainstay of treatment is surgical removal of the cancer and elimination of possible disease foci with radiation, chemotherapy, hormonal therapy, or a combination of the above $[1,2]$. The type of mastectomy that is performed depends on multiple factors, such as the size and location of the tumor, tumor response to induction chemotherapy, the size of the patient's breast compared with the cancer, and patient desires [2]. For patients with early-stage disease, breast-conserving therapies (BCT), including partial mastectomy/lumpectomy, coupled with local radiation, have been favored over total mastectomy. This approach has been shown to have equal survival rates as total mastectomy with improved aesthetic results for the breast [2-4].

Regardless of the type of mastectomy, the goal of breast reconstruction is to recreate the form and symmetry of the patient's breasts so that her body image is restored and she is satisfied with the overall appearance of her breasts. It is critical that the breast surgeon, reconstructive surgeon, and patient collectively discuss reconstructive goals and how these can be achieved after the patient's oncologic surgery.

\section{General Considerations}

Breast reconstruction has been shown to have a positive impact on quality of life following mastectomy. It has been proven to be safe and is not associated with an increased risk of cancer recurrence nor does reconstruction interfere with detection of recurrence [5-13, 14•]. Advanced-stage breast cancer is not a contraindication to breast reconstruction after mastectomy; several studies have shown reconstruction to be safe and effective for these patients $[15,16]$. Despite this evidence, rates of immediate breast reconstruction are lower than expected and range in the literature from 10-40\% $[17-21,22 \bullet, 23 \bullet, 24]$. It is important that patients are given the option to discuss breast reconstruction as part of their preoperative planning [25•]. 
The need for postoperative radiation therapy is a relative contraindication for immediate breast reconstruction using certain techniques. Patients with significant comorbidities who are not good surgical candidate typically do not undergo reconstruction. Another relative contraindication to breast reconstruction is unrealistic patient expectations. Thus, an important component of the consultation and evaluation process is addressing patient desires and expectations. Patient support groups and pre/postoperative photos will help the patient understand the various options.

\section{Reconstructive Options}

Numerous reconstructive options exist and include implant, autologous tissue flaps, or a combination of the two. Choice of the most appropriate reconstructive option depends on what is being matched with the opposite breast, patient comorbidities, the patient's body habitus, and often the surgeons' preference [26, 27•, 28-31]. Many patients will be good candidates for several different techniques, and in some scenarios, a combination of these techniques may be used to recreate the breast after mastectomy.

\section{Implant-Based Reconstruction}

Implant-based reconstruction often is preferred in that it eliminates the potential for donor site morbidity and has a quicker recovery. Younger patients and bilateral defects are more commonly reconstructed using implants (Fig. 1). These devices are typically placed beneath the pectoralis major muscle and are filled with saline or silicone gel [32]. The prosthesis-based approach requires that the patient have enough healthy tissue to cover the implant safely without excessive tension on the overlying skin. With the increasing use of skin-sparing mastectomy and nipplesparing mastectomy in the management of patients with early-stage breast cancer, this type of reconstruction has become more popular. If patients do not have adequate tissue coverage after mastectomy, a tissue-expander can be used to stretch the skin envelope gradually before placement of a prosthetic implant as a "two-stage" approach [32]. This approach using a tissue expander initially and then a few months later replacing it with the definitive implant is more common and allows revision in size and shape at the secondary procedure [31, 33-36]. Acellular dermal matrices (ADM) are used to control the expander pocket, position the pectoralis muscle, and provide coverage in the lower pole. Autologous flaps, such as the latissimus dorsi, often are performed in conjunction with expander reconstruction, especially in patients with poor skin quality, to improve coverage over the prosthesis [31].

The basic surgical technique for implant-based reconstruction after mastectomy involves the creation of a submuscular pocket that will cover the implant. This pocket is created either by elevating the pectoralis major muscle and reattaching its inferior edge to the inferior mastectomy skin flap (dual plan with Marrionette sutures) or an acellular dermal matrix sling (dual plane with ADM). The third option is total muscular coverage by creating a complete submuscular-subfascial pocket. This is performed by elevating the pectoralis major muscle from lateral to medial, raising the medial border of the serratus anterior muscle, and extending this submuscular pocket slightly below the inframammary fold by raising a small amount of anterior rectus sheath. The implant or tissue expander is then positioned within the pocket and secured.

When deciding on the best prosthesis to use, both saline and silicone implants are safe; silicone implants are not associated with increased risks of breast cancer, systemic diseases, such as rheumatoid arthritis and connective tissue disease, or gynecologic disorders as was once reported [37-39, 40•]. Compared with saline, silicone implants have a consistency that more closely resembles adipose tissue and the feel of the natural breast [40•].

The relative contraindications to implant-based reconstruction includes radiation to the chest wall, either before

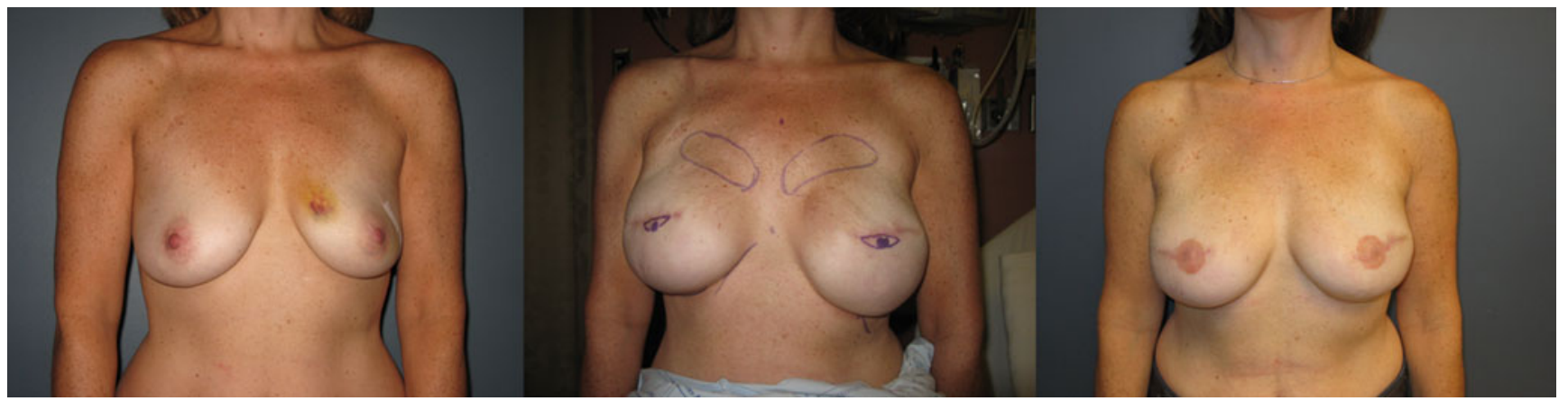

Fig. 1 A 45-year-old with a history of breast cancer underwent a bilateral skin sparing mastectomy and two-stage expander/implant reconstruction. Her result is shown 1 year after placement of $475 \mathrm{cc}$ of moderate profile plus gel-filled implants and nipple areolar reconstruction 
or after breast resection, because this has been shown to have increased complications, including implant extrusion [41-43]. Others have demonstrated that patients with hypertension, obesity, or current smoking had a higher risk of implant extrusion after reconstruction, in addition to having a higher risk of other complications after implant placement [44, 45].

The major advantages of implant-based reconstructions compared with autologous reconstructions are that the operations are shorter and potentially safer for the patient. In addition, patients are not at risk of donor-site pain or complications that can occur with autologous reconstruction procedures. Implant reconstruction is a good option for thin women who do not have adequate tissue for the flap-based reconstructions.

The disadvantages of the prosthesis-based approaches relate to the inherent risks of having foreign material housed within the breast and the number of procedures that are required to reach the final desired aesthetic outcome. Implants pose risks of infection, leakage or rupture, extrusion, and scarring/fibrosis around the implant (capsular contracture), which can cause breast deformity and may ultimately cause significant breast pain [41-43]. The use of the tissue expander requires weekly visits to the clinic to gradually fill the expander with saline before placement of the implant. Implant malfunction and size differences can occur, and patients may require additional operative procedures years after their initial reconstruction surgeries.

The ultimate goal of any reconstruction is to achieve symmetry with the opposite breast. It often is more difficult to match a larger, more ptotic breast with an implant. Contralateral breast manipulation is not uncommon, and a breast reduction or breast lift will improve symmetry in patients with large or ptotic breasts. It is not uncommon to do a contralateral breast augmentation in women with smaller breasts to improve symmetry following implant reconstruction [31]. Implant-based reconstruction often is an excellent option for patients who will undergo bilateral mastectomies following their breast cancer diagnosis, due to the inherent ease of obtaining symmetry with bilateral as opposed to unilateral reconstruction.

\section{Autologous Reconstruction}

This approach utilizes the patient's skin, underlying adipose tissue, or muscle, to recreate a natural-appearing breast, which is similar in size, consistency, and form to the patient's native breast $[27 \cdot, 28-31,46]$. The two most common donor sites include the lower abdomen and the back. Autologous tissue transfer is an excellent option for patients who have had radiation to the chest wall as part of their cancer treatment, likely due to the robust vascularity and improved propensity for healing that the tissue flap provides [47•].

Aesthetic results following autologous tissue transfer often are excellent, and this approach allows for the creation of a breast mound that closely resembles the soft, ptotic natural breast in appearance and texture. It can be particularly beneficial to women undergoing unilateral mastectomy because of the symmetry between the natural, contralateral breast and the reconstructed breast. Flap-based reconstruction also can be performed in women with bilateral mastectomies with excellent outcomes [48-50].

The advantages of autologous tissue reconstruction include the transfer of vascularized skin, muscle, and fat to provide coverage and volume, which is especially beneficial in previously irradiated patients. When this eliminates the need for implants, foreign body-related complications are eliminated and fewer procedures are typically required to complete the process.

The disadvantages of autologous reconstruction include potential donor-site morbidity and the risk of flap loss. Complications can include chronic pain, asymmetry, reduced function, and abdominal wall complications, such as hernia/bulges or weakness [46]. The procedures tend to be longer, with longer recovery times. These potential complications need to be taken into consideration when choosing the most appropriate procedure for any given patient. The importance of patient selection and risk stratification cannot be understated.

The success of the autologous tissue flap depends on the ability to retain perfusion of the transferred tissue, and there are two strategies that can be used to maintain the blood supply. The first approach is to harvest the flap without disrupting its native vascular supply and carefully dissecting out the vessels leading to the flap as a vascular "pedicle" [27•, 28-31]. The flap is then tunneled to the defect under the skin and subcutaneous adipose tissue and positioned and secured to the chest wall to create a breast mound. The pedicled flap must be harvested from sites in close proximity to the chest wall defect, and the lower abdomen and upper back are the donor sites for these pedicled flaps, i.e., the transverse rectus abdominis myocutaneous (TRAM) flap based on the superior epigastric vessels [51] and the latissimus dorsi myocutaneous flap based on the thoracodorsal vessels [52].

Alternatively, "free" tissue flaps can be harvested from a variety of donor sites. This approach involves identifying, isolating, and ligating the vessels that supply the desired skin, subcutaneous adipose tissue, and muscle [46]. The flap is then transferred to the fill the chest wall defect and anastomosed to recipient vessels in proximity to the defect using microsurgical techniques. The choice of the recipient vessels depends on the anatomy present after the mastectomy. The internal mammary or thoracodorsal vessels are 
more commonly used. The ability to use free tissue flaps for breast reconstruction expands the potential donor sites available to the patient, which is important for patients who have had prior surgeries that may have affected the vascular supply to a given flap. Depending on a patient's body habitus, certain donor sites are inherently better suited than others to provide adequate subcutaneous tissue and volume for the transferred flap. The main free flaps that are used for breast reconstruction include the free TRAM, the DIEP (deep inferior epigastric perforator), and SIEA (superficial inferior epigastric artery) flaps, all of which are based on the inferior epigastric vessels of the lower abdomen. Additional flaps include the Rubens flap, based on the deep circumflex iliac pedicle, the ALT (anterior lateral thigh) flap, based on the descending branch of lateral femoral circumflex artery, the TUG (transverse upper gracilis) flap, supplied by the medial femoral cutaneous artery, and the gluteal flaps, based either on the superior or inferior gluteal arteries and veins.

\section{Abdominal Flap Options}

The abdomen typically has adequate skin and subcutaneous tissue to provide coverage and volume replacement after mastectomy. Additionally, harvesting of the abdominal wall tissue is performed in a manner similar to a cosmetic abdominoplasty, and many patients are pleased with this additional aesthetic benefit. The pedicled and free TRAM flaps are considered by many plastic surgeons to be the firstline flaps for breast reconstruction, because they have a predictable vascular anatomy, can be reliably harvested, and have excellent aesthetic outcomes (Fig. 2) [46, 51,
53]. The free TRAM is particularly advantageous over its pedicled counterpart, because it is based on the inferior epigastric vessels, which provide a more robust blood supply to the transferred flap and therefore reduce the risk of partial flap loss.

The TRAM flaps tend to be plagued by the postoperative development of abdominal hernias, as a result of the weakened abdominal wall after harvesting of the rectus abdominis [31, 51]. Recent improvements have led to the development of the muscle-sparing TRAM and perforatorbased DIEP- and SIEA-free flaps, which minimize or eliminate the harvesting of muscle at the donor site, thus decreasing donor site complications compared with the TRAM flap [54, 55].

\section{Latissimus Dorsi Flap}

Unlike the abdomen, the back usually does not have sufficient subcutaneous tissue required to recreate the breast mound with a latissimus myocutaneous flap alone, and this flap most often is used as a combination approach along with tissue expanders/implants to reconstruct the breast (Fig. 3). The main advantages of this type of reconstruction include the reliability and predictability of the flap and the absence of significant donor-site morbidity even in high-risk patients [56-58]. The most common complication is the development of a donor site seroma in almost $50 \%$ of patients. The latissimus dorsi is a useful option in obese patients and an extended autologous flap can be used without an implant to match a decent-sized contralateral breast in certain patients.
Fig. 2 A 58-year-old woman underwent a left skin-sparing mastectomy with a latissimus dorsi reconstruction with an implant. Her shape and symmetry is shown at 1 year after nipple areolar reconstruction

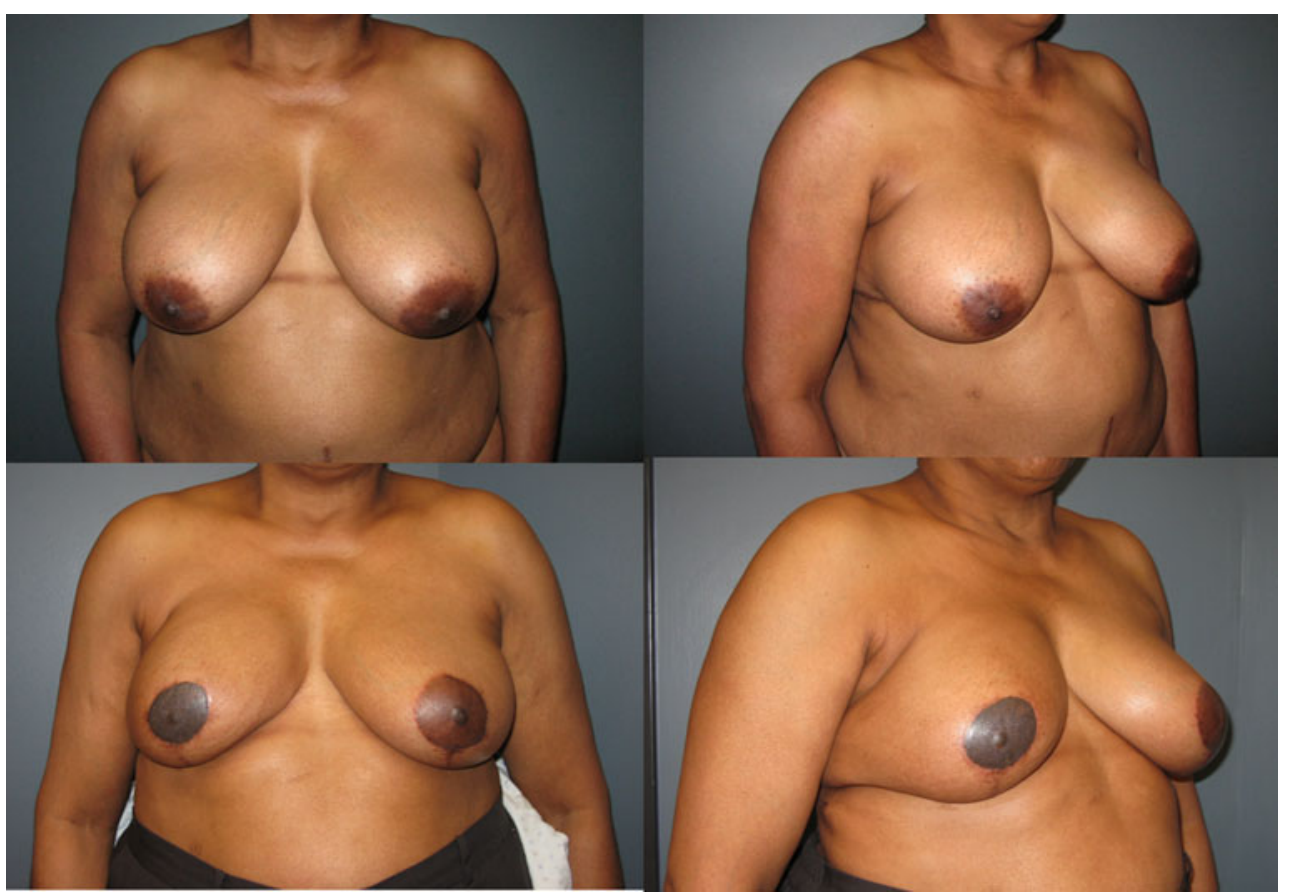


Fig. 3 A 64-year-old woman with moderate ptosis underwent a mastectomy and TRAM flap reconstruction. A contralateral reduction was performed for symmetry and the TRAM flap was able to match a natural opposite breast

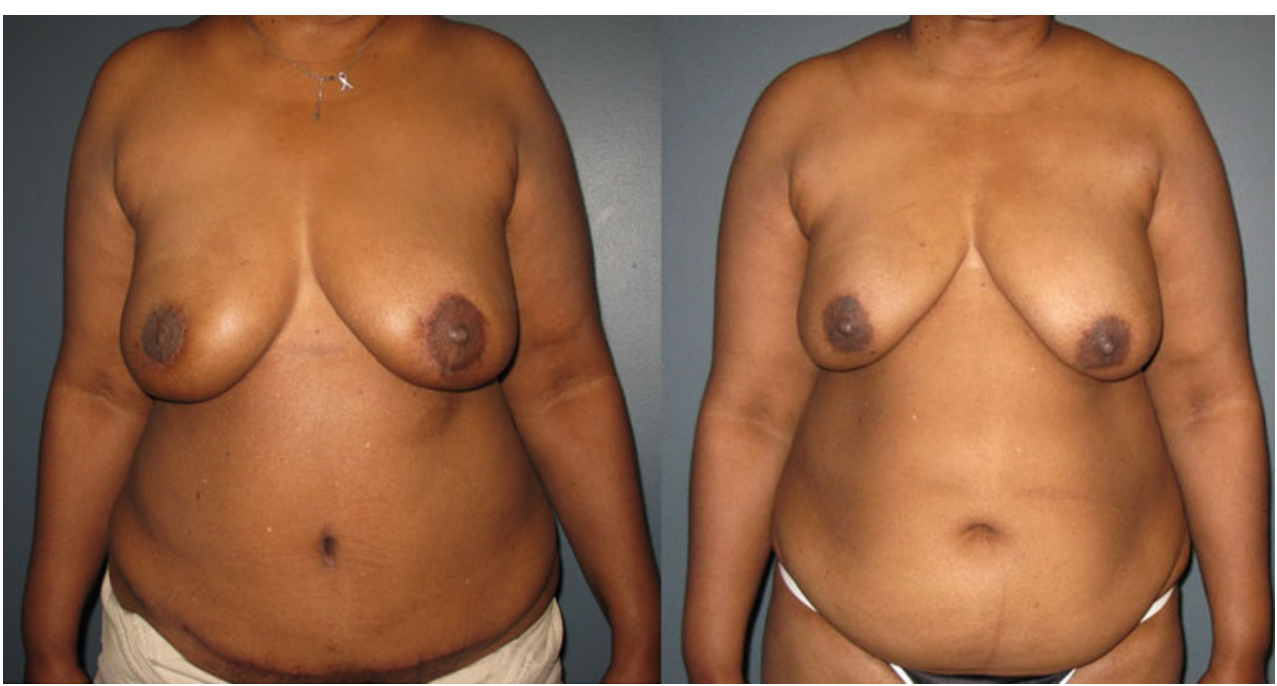

\section{Other Flaps}

Whereas the abdomen and upper back are the most common donor sites, other free flap options include the gluteal flaps (SGAP, IGAP), TUG flap, and Rubens flap. These flaps are generally considered when patients are not candidates for the abdominal-based or latissimus dorsi flaps. The gluteal flaps are advantageous, because most patients will have tissue available for transfer; however, these are technically challenging flaps to raise and donor site deformity can be significant. The Rubens flap contains the fatty region generally described as the hip and is based on the deep circumflex iliac vessels. The closure of the abdominal wall defect after flap harvest can be challenging and probably limits the use of this particular approach. The TUG flap uses tissue from the inner thigh and is usually used for patients who have had previous abdominal surgery, inadequate skin or tissue at the other donor sites, or for those that desire a "thigh lift" as an additional component of their reconstructive procedure. These flaps are all considered tertiary types of breast reconstruction.

\section{When to Reconstruct?}

The timing of breast reconstruction is either at the time of mastectomy (immediate) or at a later stage (delayed). The decision depends on individual patient characteristics, whether or not the patient will be treated with radiation, as well as the preferences of both the patient and surgeons [59]. In general, immediate reconstruction with skin-sparing mastectomy is safe and results in improved patient psyche and aesthetic outcomes. Delayed reconstruction is indicated if patients will require postmastectomy radiation as part of their cancer treatment, if patients do not have access to reconstruction for scheduling issues, or if the patient is unable to tolerate a long procedure at that time. Several reports have indicated that it is best to delay breast reconstruction until radiation therapy is complete, because the presence of the reconstructed breast can interfere with radiation efficiency and the ability to target the chest wall [47•]. In addition, radiation to the reconstructed breast results in tissue damage, disfigurement, and can precipitate complications in both flap-based and implant-based reconstructions [60]. It is preferred to defer autologous abdominal tissue reconstruction until completion of radiation therapy. In general, reconstruction often is delayed for 6-12 months after radiation therapy is complete to allow tissue healing and to appreciate fully the extent of postradiation damage to the tissue. Alternatively, tissue expanders can be placed at the time of mastectomy to maintain the skin envelope during radiation therapy, and the reconstruction delayed until after radiation is complete as a "delayed-immediate" approach [47•,61]. This also preserves the option of implant reconstruction if the skin envelope is favorable, especially for younger patients with smaller breasts and bilateral mastectomy defects.

\section{Reconstructing the Partial Mastectomy Defect}

The option to reconstruct partial mastectomy defects in patients who undergo breast conservation therapy (BCT) also has become more popular recently [26, 29, 62•, 63-65]. This is performed in an attempt to maximize the amount of tissue resected and minimize the potential for a poor cosmetic result. The two main options are local tissue rearrangement, such as reduction and mastopexy techniques or a local flap. This often is referred to as the oncoplastic approach and is designed to achieve complete oncologic resection while retaining the aesthetic appearance of the breast. The oncoplastic reduction techniques are more common in this country, because BCT in patients with macromastia often will result in radiation dosing inhomogeneity 
and poor cosmesis. The benefits are that resections will be more generous; additional tissue is typically removed with the potential advantage of fewer positive margins and subsequently lower recurrence rates. It also allows reduction of the opposite breast with tissue sampling, and the benefits of smaller breasts for women with symptomatic macromastia. Careful planning of the lines of surgical resection allow the tumor to be removed along with the excess breast tissue and local tissue rearrangements within the breast mound fill the defect after resection [63].

Oncoplastic reduction combined with radiation has been shown to be oncologically safe with improvement in the appearance of the breast after resection [64, 66, 67]. The type of reduction technique depends on the size and location of the tumor within the breast and the size of the breast itself. Patients with large, pendulous breasts are usually the best candidates for this approach, although oncoplastic reduction has been successful in some women with small- to medium-size breasts and comparatively smaller tumor volumes [68]. Additionally, induction chemotherapy can be used to shrink the tumor before resection to improve tumor:breast volume ratios [69]. As with other BCTs, all patients opting for oncoplastic reduction as a treatment for their breast cancer also will require radiation therapy after resection to improve oncologic outcomes $[65,70]$. Therefore, this procedure may not be an option for pregnant patients or women who have had prior radiation to the same chest wall and therefore are poor candidates for further radiation therapy. Women with smaller breasts who do not have enough tissue remaining following the lumpectomy to reshape the mound often will require local flap reconstruction of their partial mastectomy defect if a poor cosmetic result is anticipated. The latissimus dorsi flap is a workhorse flap for this indication but is at the expense of using up a potential reconstructive option if completion mastectomy is ever required. The potential for positive margins should always be discussed with the patient and needs to be taken into consideration when choosing and designing the partial breast reconstructive technique.

\section{Conclusions}

There are many reconstructive options available to women who will require partial or total mastectomy as a component of their breast cancer therapy. The patient will ideally learn about her reconstructive options before undergoing mastectomy, and conversations among the patient, breast surgeon, and reconstructive surgeon will guide the decisions related to the type of mastectomy and the type of reconstruction that will give the best functional and aesthetic outcomes for the patient.
Disclosure No potential conflicts of interest relevant to this article were reported.

\section{References}

Papers of particular interest, published recently, have been highlighted as:

- Of importance

1. ACS, American Cancer Society: Breast Cancer Facts \& Figures 2011-2012. 2011.

2. Maughan KL, Lutterbie MA, Ham PS. Treatment of breast cancer. Am Fam Physician. 2010;81(11):1339-46.

3. Clarke M, et al. Effects of radiotherapy and of differences in the extent of surgery for early breast cancer on local recurrence and 15-year survival: an overview of the randomised trials. Lancet. 2005;366(9503):2087-106.

4. Fisher B, et al. Five-year results of a randomized clinical trial comparing total mastectomy and segmental mastectomy with or without radiation in the treatment of breast cancer. N Engl J Med. 1985;312(11):665-73.

5. Murphy Jr RX, et al. Impact of immediate reconstruction on the local recurrence of breast cancer after mastectomy. Ann Plast Surg. 2003;50(4):333-8.

6. Vandeweyer E, et al. Immediate breast reconstruction with salinefilled implants: no interference with the oncologic outcome? Plast Reconstr Surg. 2001;107(6):1409-12.

7. McCarthy CM, et al. Breast cancer recurrence following prosthetic, postmastectomy reconstruction: incidence, detection, and treatment. Plast Reconstr Surg. 2008;121(2):381-8.

8. Meretoja TJ, et al. Local recurrence of stage 1 and 2 breast cancer after skin-sparing mastectomy and immediate breast reconstruction in a 15-year series. Eur J Surg Oncol. 2007;33(10):1142-5.

9. Munhoz AM, et al. Immediate skin-sparing mastectomy reconstruction with deep inferior epigastric perforator (DIEP) flap. Technical aspects and outcome. Breast J. 2007;13(5):470-8.

10. Langstein $\mathrm{HN}$, et al. Breast cancer recurrence after immediate reconstruction: patterns and significance. Plast Reconstr Surg. 2003;111(2):712-20. discussion 721-2.

11. Rinker BD, Bowling JT, Vasconez HC. Blood transfusion and risk of metastatic disease or recurrence in patients undergoing immediate TRAM flap breast reconstruction: a clinical study and metaanalysis. Plast Reconstr Surg. 2007;119(7):2001-7.

12. Vaughan A, et al. Scientific presentation award. Patterns of local breast cancer recurrence after skin-sparing mastectomy and immediate breast reconstruction. Am J Surg. 2007;194 (4):438-43.

13. Huang CJ, et al. Comparison of local recurrence and distant metastases between breast cancer patients after postmastectomy radiotherapy with and without immediate TRAM flap reconstruction. Plast Reconstr Surg. 2006;118(5):1079-86. discussion 1087-8.

14. - Losken A, et al. The impact of partial breast reconstruction using reduction techniques on postoperative cancer surveillance. Plast Reconstr Surg. 2009;124(1):9-17. This study compared postoperative surveillance in patients with oncoplastic reduction and standard breast-conserving therapies and found that there is no difference in recurrance between the two groups however oncoplastic reduction does have a longer time to mammographic stability.

15. Crisera CA, et al. Immediate free flap reconstruction for advancedstage breast cancer: is it safe? Plast Reconstr Surg. 2011;128 (1):32-41. 
16. Nahabedian MY. Discussion: immediate free flap reconstruction of advanced-stage breast cancer: is it safe? Plast Reconstr Surg. 2011;128(1):42-3.

17. Fallbjork U, et al. Differences between women who have and have not undergone breast reconstruction after mastectomy due to breast cancer. Acta Oncol. 2010;49(2):174-9.

18. Finlayson CA, MacDermott TA, Arya J. Can specific preoperative counseling increase the likelihood a woman will choose postmastectomy breast reconstruction? Am J Surg. 2001;182 (6):649-53.

19. Reaby LL. Reasons why women who have mastectomy decide to have or not to have breast reconstruction. Plast Reconstr Surg. 1998;101(7):1810-8.

20. Agarwal S, et al. Survival in breast cancer patients undergoing immediate breast reconstruction. Breast J. 2010;16(5):503-9.

21. Rosson GD, et al. Multilevel analysis of the impact of community vs patient factors on access to immediate breast reconstruction following mastectomy in Maryland. Arch Surg. 2008;143(11):107681. discusion 1081.

22. - Agarwal S, et al. An analysis of immediate postmastectomy breast reconstruction frequency using the surveillance, epidemiology, and end results database. Breast J. 2011;17(4):352-8. This study by Agarwal et al. is of interest to breast cancer patients and their providers as it examines the patient factors that are associated with the decision to undergo breast reconstruction.

23. Alderman AK, et al. Racial and ethnic disparities in the use of postmastectomy breast reconstruction: results from a populationbased study. J Clin Oncol. 2009;27(32):5325-30. Alderman et al. have specifically examined the issue of breast reconstruction in minority women and the factors that influence the decision to reconstruct and/or access to care. This study examined specifically the Latina demographic, in addition to other patient populations.

24. Reuben BC, Manwaring J, Neumayer LA. Recent trends and predictors in immediate breast reconstruction after mastectomy in the United States. Am J Surg. 2009;198(2):237-43.

25. - Preminger BA, et al. Improving access to care: breast surgeons, the gatekeepers to breast reconstruction. J Am Coll Surg. 2012;214 (3):270-6. Recent work by Preminger et al. examining access to care. This study further supported the influence of breast surgeons in breast cancer patients' reconstructive process.

26. Losken A, Ghazi B. An update on oncoplastic surgery. Plast Reconstr Surg. 2012;129(2):382e-3e.

27. - Roostaeian J, Crisera C. Current options in breast reconstruction with or without radiotherapy. Curr Opin Obstet Gynecol. 2011;23 (1):44-50. This is an excellent review on breast reconstruction and addresses the concerns regarding radiation in detail.

28. Djohan R, Gage E, Bernard S. Breast reconstruction options following mastectomy. Cleve Clin J Med. 2008;75 Suppl 1: S17-23.

29. Urban C, et al. Oncoplastic principles in breast conserving surgery. Breast. 2011;20 Suppl 3:S92-5.

30. Serletti JM, et al. Breast reconstruction after breast cancer. Plast Reconstr Surg. 2011;127(6):124e-35e.

31. Cordeiro PG. Breast reconstruction after surgery for breast cancer. N Engl J Med. 2008;359(15):1590-601.

32. Spear SL, Mesbahi AN. Implant-based reconstruction. Clin Plast Surg. 2007;34(1):63-73. abstract vi.

33. Roostaeian J, et al. Comparison of immediate implant placement versus the staged tissue expander technique in breast reconstruction. Plast Reconstr Surg. 2012;129(6):909e-18e.

34. Bailey $\mathrm{MH}$, et al. Immediate breast reconstruction: reducing the risks. Plast Reconstr Surg. 1989;83(5):845-51.

35. Eriksen $\mathrm{C}$, et al. A prospective randomized study comparing two different expander approaches in implant-based breast reconstruction: one stage versus two stages. Plast Reconstr Surg. 2012;130 (2):254e-64e.
36. Settembrini F, Bonomi S, Gregorelli C. Patient selection and aesthetic outcomes in one-stage versus two-stage implant breast reconstruction. Plast Reconstr Surg. 2012;129(2):364e-6e.

37. Brody GS. On the safety of breast implants. Plast Reconstr Surg. 1997;100(5):1314-21.

38. Holmich LR, et al. Breast implant rupture and connective tissue disease: a review of the literature. Plast Reconstr Surg. 1997;120(7 Suppl 1):62S-9S.

39. Lewin SL, Miller TA. A review of epidemiologic studies analyzing the relationship between breast implants and connective tissue diseases. Plast Reconstr Surg. 1997;100(5):1309-13.

40. - Spear SL, Jespersen MR. Breast implants: saline or silicone? Aesthetic Surg J. 2010;30(4):557-70. Another excellent and recent review that discusses the current state of breast implants, which is of interest to patients and their providers.

41. Cordeiro PG, McCarthy CM. A single surgeon's 12-year experience with tissue expander/implant breast reconstruction: part II. An analysis of long-term complications, aesthetic outcomes, and patient satisfaction. Plast Reconstr Surg. 2006;118(4):832-9.

42. Cordeiro PG, McCarthy CM. A single surgeon's 12-year experience with tissue expander/implant breast reconstruction: part I. A prospective analysis of early complications. Plast Reconstr Surg. 2006;118(4):825-31.

43. Alderman AK, et al. Complications in postmastectomy breast reconstruction: two-year results of the Michigan breast reconstruction outcome study. Plast Reconstr Surg. 2002;109(7):2265-74.

44. Vega SJ, et al. Gracilis myocutaneous free flap in autologous breast reconstruction. Plast Reconstr Surg. 2009;124(5):1400-9.

45. McCarthy CM, et al. Predicting complications following expander/implant breast reconstruction: an outcomes analysis based on preoperative clinical risk. Plast Reconstr Surg. 2008;121 (6):1886-92.

46. Rosson GD, et al. A review of the surgical management of breast cancer: plastic reconstructive techniques and timing implications. Ann Surg Oncol. 2010;17(7):1890-900.

47. - Kronowitz SJ, Robb GL. Radiation therapy and breast reconstruction: a critical review of the literature. Plast Reconstr Surg. 2009;124(2):395-408. Kronowitz et al. highlight the management decisions that must be made in breast cancer patients that will receive or may receive chest wall radiation as a component of therapy. Also an excellent discussion regarding the use of the "delayed-immediate" approach to reconstruction.

48. Losken A, et al. Outcomes evaluation following bilateral breast reconstruction using latissimus dorsi myocutaneous flaps. Ann Plast Surg. 2010;65(1):17-22.

49. Werdin F, et al. Superior gluteal artery perforator flap in bilateral breast reconstruction. Ann Plast Surg. 2010;64(1):17-21.

50. Dao TN, Verheyden CN. TRAM flaps: a reconstructive option after bilateral nipple-sparing total mastectomy. Plast Reconstr Surg. 2005;116(4):986-92.

51. Jones G. The pedicled TRAM flap in breast reconstruction. Clin Plast Surg. 2007;34(1):83-104. abstract vii.

52. Hammond DC. Latissimus dorsi flap breast reconstruction. Clin Plast Surg. 2007;34(1):75-82. abstract vi-vii.

53. Tachi M, Yamada A. Choice of flaps for breast reconstruction. Int $\mathrm{J}$ Clin Oncol. 2005;10(5):289-97.

54. Chen CM, et al. Immediate postoperative complications in DIEP versus free/muscle-sparing TRAM flaps. Plast Reconstr Surg. 2007;120(6):1477-82.

55. Nahabedian MY, et al. Breast Reconstruction with the free TRAM or DIEP flap: patient selection, choice of flap, and outcome. Plast Reconstr Surg. 2002;110(2):466-75. discussion 476-7.

56. Laitung JK, Peck F. Shoulder function following the loss of the latissimus dorsi muscle. Br J Plast Surg. 1985;38(3):375-9.

57. Russell RC, et al. Functional evaluation of latissimus dorsi donor site. Plast Reconstr Surg. 1986;78(3):336-44. 
58. Fraulin FO, et al. Functional evaluation of the shoulder following latissimus dorsi muscle transfer. Ann Plast Surg. 1995;35 (4):349-55.

59. Ananthakrishnan P, Lucas A. Options and considerations in the timing of breast reconstruction after mastectomy. Cleve Clin $\mathrm{J}$ Med. 2008;75 Suppl 1:S30-3.

60. Barry M, Kell MR. Radiotherapy and breast reconstruction: a meta-analysis. Breast Canc Res Treat. 2011;127(1):15-22.

61. Peled AW, et al. Increasing the time to expander-implant exchange after postmastectomy radiation therapy reduces expander-implant failure. Plast Reconstr Surg. 2012;130(3):503-9.

62. - Losken A, Hamdi M. Partial breast reconstruction: current perspectives. Plast Reconstr Surg. 2009;124(3):722-36. This recent review highlights partial breast reconstruction and further discusses the use of oncoplastic reducation as an option for patients with breast cancer.

63. Iwuchukwu OC, et al. The role of oncoplastic therapeutic mammoplasty in breast cancer surgery-a review. Surg Oncol. 2012;21 (2): $133-41$.

64. Fitoussi AD, et al. Oncoplastic breast surgery for cancer: analysis of 540 consecutive cases [outcomes article]. Plast Reconstr Surg. 2010;125(2):454-62.
65. Fisher B, et al. Twenty-year follow-up of a randomized trial comparing total mastectomy, lumpectomy, and lumpectomy plus irradiation for the treatment of invasive breast cancer. N Engl J Med. 2002;347(16):1233-41.

66. Song HM, et al. The use of oncoplastic reduction techniques to reconstruct partial mastectomy defects in women with ductal carcinoma in situ. Breast J. 2010;16(2):141-6.

67. Chakravorty A, et al. How safe is oncoplastic breast conservation? Comparative analysis with standard breast conserving surgery. Eur J Surg Oncol. 2012;38(5):395-8.

68. Yang JD, et al. Surgical techniques for personalized oncoplastic surgery in breast cancer patients with small- to moderate-sized breasts (part 1): volume displacement. J Breast Canc. 2012;15 (1):1-6.

69. Grubnik, A., C. Benn, and G. Edwards, Therapeutic Mammaplasty for Breast Cancer: Oncological and Aesthetic Outcomes. World J Surg, 2012.

70. Mahmood U, et al. Similar survival with breast conservation therapy or mastectomy in the management of young women with early-stage breast cancer. Int J Radiat Oncol Biol Phys. 2012;83 (5):1387-93. 